\title{
Near-Surface Vortex Formation in Supercells from the Perspective of Vortex Patch Dynamics
}

\author{
JOHANNES M. L. DAHL \\ Department of Geosciences, Texas Tech University, Lubbock, Texas
}

(Manuscript received 9 March 2020, in final form 22 May 2020)

\begin{abstract}
In many supercell simulations, near-ground vortex formation results from the collapse of an elongated region of enhanced vertical vorticity. In this study, this "roll-up" mechanism is analyzed by investigating the behavior of several 2D elliptic vortex patches. The problem is treated as a nonlinear initial value problem, which is better suited to describe the roll-up mechanism than the more commonly employed normal-mode analysis. Using the Bryan Cloud Model 1, it is demonstrated that the condition for vortex formation is an initial finite-amplitude nonuniformity within the vortex patch. Vortex formation results from differential self-advection due to the flow induced by the patch itself. Background straining motion may either aid or suppress vortex-patch axisymmetrization depending on the initial orientation of the patch relative to the deformation axis. It is also found that in some cases numerical dispersion may lead to nonuniformities that serve as seed for axisymmetrization, thus resulting in unphysical vortex development.
\end{abstract}

\section{Introduction}

Tornadoes and tornado-like vortices in supercell simulations often develop out of elongated regions of vertical vorticity (called "vertical-vorticity rivers" by Dahl et al. 2014), that collapse into a vortex (Gaudet and Cotton 2006; Gaudet et al. 2006; Markowski et al. 2014). These regions of enhanced vertical vorticity may be approximated as vortex patches (i.e., finite areas of nonzero vertical vorticity surrounded by irrotational fluid) (Saffman 1992, p. 160). (The reader may skip forward to Fig. 7a for an example of such an elongated vortex patch.) It is well established that the initial appearance of weak near-surface vertical vorticity extrema, or vortex patches, occurs within the cold pool of the thunderstorm (Rotunno and Klemp 1985; DaviesJones and Brooks 1993; Markowski and Richardson 2014; Dahl et al. 2014; Parker and Dahl 2015; Dahl 2015). The vortex patches result mainly from baroclinically produced, horizontal vorticity that is tilted into the vertical, as demonstrated by Klemp and Rotunno (1983) and Rotunno and Klemp (1985). This mechanism was further elucidated by Davies-Jones and Brooks (1993, hereafter DJB93), who showed that horizontal baroclinic vorticity production, and subsequent tilting into

Corresponding author: Johannes Dahl, johannes.dahl@ttu.edu the vertical, occurs in descending air leading to a vertical vorticity component at the surface (DJB93; Dahl et al. 2014; Markowski and Richardson 2014; Dahl 2015; Parker and Dahl 2015). This vertical vorticity tends to be accumulated along convergence boundaries within and at the edge of the cold pool, whereby elongated vortex patches result (Markowski et al. 2014).

Once such an elongated vortex patch is present, the question arises by what mechanism it may collapse into a compact vortex. These elongated vortex patches have been noted in numerous more or less idealized supercell simulations (Dahl et al. 2014; Markowski et al. 2014; Nowotarski et al. 2015; Parker and Dahl 2015; Coffer and Parker 2017). In a recent very high-resolution simulation of a tornadic supercell, Orf et al. (2017) similarly identified a band of vertical vorticity along the forwardflank gust front, although the structure of this band was much more complex than in lower-resolution simulations, containing a train of oppositely signed vortices. Despite their ubiquity, the behavior of these vorticity bands is rarely discussed. Instead, the focus is almost exclusively on analyzing the mechanisms leading to large near-ground vertical vorticity or circulation (e.g., Rotunno and Klemp 1985; Wicker and Wilhelmson 1995; Dahl et al. 2014; Markowski and Richardson 2014; Schenkman et al. 2014; Mashiko 2016; Roberts and Xue 2017; Flournoy and Coniglio 2019; Tao and Tamura 2020, 
among others). The only exception the author is aware of is the study by Gaudet et al. (2006), in which the collapse of a vorticity band into a vortex in a simulated supercell is carefully analyzed. In that study, vortex formation is discussed in the context of the growth of unstable normal modes, and different theoretical models to explain subsequent vortex formation are presented.

In the context of nonmesocyclonic tornadoes, vortex sheet instability has been discussed by Lee and Wilhelmson (1997), who found initial growth consistent with unstable normal modes. Because the most unstable mode dominates after some time, a rather regularly spaced string of like-signed vortices arises, with vortex formation following nonlinear growth and interactions between the unstable modes (Lee and Wilhelmson 1997).

As exemplified by these studies the common approach to understanding the initiation of vortex development within a shear layer is based on linear normal-mode stability analysis. In simulated supercells, however, mesocyclonic tornado-like vortices (TLVs) do not seem to develop out of a string of likesigned vortices that would result from growing normal modes. Moreover, instead of a vortex sheet the vertical vorticity often appears as more or less elongated, sometimes rather amorphous, nonuniform vortex patch which evolves into an approximately symmetric vortex as a whole. In this study vortex formation near the surface in supercells is thus explored in terms of an initial value problem instead of a normal-mode analysis. The problem will be treated as vortex-patch axisymmetrization (e.g., Melander et al. 1987), and several 2D simulations will be presented to illustrate the process. The purpose of this paper is to demonstrate that the basic mechanism leading to vortex formation may simply be understood via differential self-advection of the vortex patches, consistent with the findings by Gaudet et al. (2006). In doing so, we seek to apply concepts borrowed from basic fluid physics to vorticity structures observed in simulated supercells (rather than presenting fundamentally new concepts regarding vortex formation).

The relevance of vortex-patch axisymmetrization is that the central pressure in the developing vortex decreases during the process, which may lead to rapid vortex intensification (Markowski and Richardson 2014). In particular, if a strong pressure deficit is present in the vortex center, near-surface horizontal vorticity may be stretched horizontally and then tilted upward directly at the base of the vortex, as observed in numerical simulations by, for example, Lewellen and Lewellen (2007),
Schenkman et al. (2014), Roberts and Xue (2017), Mashiko (2016), Rotunno et al. (2017), Tao and Tamura (2020), and Boyer and Dahl (2020, manuscript submitted to Mon. Wea. Rev.).

The remainder of the paper is structured as follows. In the next section, limitations of the traditional shear instability analysis in terms of normal modes are discussed, and the initial value approach will be introduced. After the methods are introduced in section 3, section 4 presents results of the $2 \mathrm{D}$ simulations, which are discussed in section 5. Conclusions are offered in section 6 .

\section{Background}

Discrete normal modes are very helpful in assessing the stability of a given flow because they are comparatively simple to analyze. One usually assumes a homogeneous base state and obtains linear equations for the perturbations (e.g., Drazin and Reid 1981). In the $(x, y)$ plane the perturbations $\Phi^{\prime}(x, y, t)$ are described as normal modes:

$$
\Phi^{\prime}(x, y, t)=\hat{\Phi}(y) e^{i(k x-c t)},
$$

where $\hat{\Phi}(y)$ is the complex amplitude, $k$ is the wavenumber, and $c$ is the complex phase speed. This treatment leads to an eigenvector problem with the phase speeds as eigenvalues. Instability exists if there are eigenvalues with positive imaginary parts (e.g., Drazin and Reid 1981, p. 128). Aside from the fact that shear instability analysis only captures the linear part of the problem, the perhaps bigger issue is that in general, arbitrary localized disturbances and their evolution cannot be described with this approach. The reason is that the set of normal modes is usually incomplete, meaning that for each wavenumber there is only a finite number of allowable phase speeds (Drazin and Reid 1981, p. 129). ${ }^{1}$ However, to describe the evolution of a localized disturbance, all wavelengths need to be able to grow and propagate at arbitrary rates. Otherwise after some time the most unstable wavelength dominates and the disturbance disperses. Thus, given their incompleteness, discrete normal modes are suitable for describing growing corrugations but not the symmetrization of a vortex patch. To address this shortcoming, an initial value problem may be formulated, whereby the discrete modes are augmented by a continuous spectrum,

\footnotetext{
${ }^{1}$ An extreme example of incompleteness is the plane Couette flow for which the spectrum of discrete normal modes is empty [however, there exists a continuous spectrum which may lead to algebraic growth, e.g., Case (1960), Farrell (1982)].
} 
which may exhibit transient nonmodal (algebraic) growth that differs from the exponential normal-mode growth. These aspects of perturbations in sheared flows are discussed in detail by, for example, Case (1960), Pedlosky (1964), Drazin and Reid (1981), Farrell (1982, 1984, 1987), or Berger (1988). The mathematical treatment of nonmodal growth is rather involved, and a perhaps more straightforward solution of the initial value problem is a numerical approach, which is pursued herein. In this treatment there is no homogeneous base state from which perturbations grow, but the elongated vortex patch (which would represent the base state in the normal mode analysis) itself evolves into a vortex.

To gain a basic understanding of vortex axisymmetrization, we will assume a 2D setup and implement the initial value problem by prescribing an arbitrary initial vertical vorticity distribution. Since the flow fields in this setup are purely horizontal, vertical vorticity will simply be referred to as vorticity. Rather than integrating the vorticity equation, the momentum equations are solved using the Bryan Cloud Model 1 (CM1; see Bryan and Fritsch 2002 or Bryan and Morrison 2012); the model setup will be introduced in the next section. To provide the initial velocity field for CM1, the vorticity field must be inverted. A horizontal, solenoidal flow $\mathbf{v}=(u, v)$ may be described in terms of a streamfunction $\psi$ as

$$
\mathbf{v}=-\mathbf{k} \times \nabla_{h} \psi,
$$

from which it follows that

$$
\nabla_{h}^{2} \psi=-\zeta
$$

where $\nabla_{h}$ is the horizontal gradient operator and $\zeta$ is the vorticity. Assuming that the region of interest is located away from the boundaries, the Green's function solution of Eq. (3) is given by

$$
\psi(\mathbf{r})=-\frac{1}{2 \pi} \int d^{2} r^{\prime} \zeta\left(\mathbf{r}^{\prime}\right) \ln \left(\left|\mathbf{r}-\mathbf{r}^{\prime}\right|\right),
$$

where $\mathbf{r}$ is the horizontal position vector and $\mathbf{r}^{\prime}$ is the integration variable. The induced horizontal flow field due to the vorticity distribution $\zeta(\mathbf{r})$ is then given by

$$
u(x, y)=\frac{\partial \psi}{\partial y}=-\frac{1}{2 \pi} \int d^{2} r^{\prime} \frac{\zeta\left(\mathbf{r}^{\prime}\right)\left(y-y^{\prime}\right)}{\left(x-x^{\prime}\right)^{2}+\left(y-y^{\prime}\right)^{2}}
$$

and

$$
v(x, y)=-\frac{\partial \psi}{\partial x}=\frac{1}{2 \pi} \int d^{2} r^{\prime} \frac{\zeta\left(\mathbf{r}^{\prime}\right)\left(x-x^{\prime}\right)}{\left(x-x^{\prime}\right)^{2}+\left(y-y^{\prime}\right)^{2}} .
$$

These integrals are solved numerically, whereby the flow field associated with the vorticity distribution is recovered. Although this flow field is referred to as "induced" flow, there is no cause and effect, or time lag, implied. The induced flow is simply the velocity field required to match the instantaneous vorticity distribution in a given domain as well as the boundary conditions (in this case, homogeneous Dirichlet conditions are used). Calculating the flow associated with the vorticity distribution does not yield the complete velocity field, but only the nondivergent (solenoidal) part, which contains rotating and straining motion [see e.g., Lynch $(1988,1989)$ for details on the decomposition of the total flow field]. The possible effects of this limitation will be discussed in section 5 .

Because the flow is $2 \mathrm{D}$ and nondivergent, the physical mechanism of the vorticity evolution is easily understood via the vorticity equation, which is in case of horizontally homogeneous density is given by

$$
\frac{\partial \zeta}{\partial t}=-\mathbf{v} \cdot \nabla_{h} \zeta+D
$$

where $D$ incorporates the contributions due to subgridscale mixing as well as implicit diffusion. This shows that aside from a usually small amount of mixing, the local vorticity changes are due only to the redistribution of existing vorticity contours via advection by the horizontal wind, and no stretching or tilting occurs. The vorticity magnitude within a patch only changes due to the mixing terms, which usually are not very large (i.e., vorticity is largely conserved materially). Note that the velocity magnitude may change significantly during the vorticity rearrangement because the solution of a Poisson equation, such as Eq. (3), scales with the wavelength of the forcing term.

\section{Describing vortex formation}

Here vortex formation is interpreted as vortexpatch axisymmetrization, which is associated with an increase of rotational flow compared to straining motion. Schielicke et al. (2016) demonstrated the utility of Truesdell's kinematical vorticity number $W_{k}$ (e.g., Truesdell 1954, p. 107) in characterizing atmospheric vortices. In 2D the kinematical vorticity number may be written as

$$
W_{k}=\frac{\sqrt{\zeta^{2}}}{\sqrt{D_{1}^{2}+D_{2}^{2}}},
$$

where $D_{1}$ and $D_{2}$ represent horizontal shearing and stretching deformation, respectively. Divergence is 
not included in the denominator because in this analysis the flow is nondivergent. The larger $W_{k}$, the more vortical the flow. Here the kinematical vorticity number is preferred over the perhaps more commonly used Okubo-Weiss (OW) number (e.g., Markowski et al. 2011; Dahl 2017) because it better captures vortex-patch axisymmetrization. In cases with a rather nonuniform initial vorticity distribution prior to axisymmetrization, the OW number already tends to highlight the region as vortex whereas $W_{k}$ does not.

\section{Model setup}

To model the evolution of the vortex patches, release 18 of CM1 was used, with a fifth-order advection scheme without explicit diffusion and free-slip upper and lower boundary conditions. A radiation condition is used for the lateral boundaries. The model was run dry and the grid spacing in all idealized settings was $100 \mathrm{~m}$ in all directions within horizontal domains that varied between $30 \times 30 \mathrm{~km}^{2}$ and $30 \times 80 \mathrm{~km}^{2}$ depending on the geometry of the vortex patch. The number of vertical levels was set to five (which is the smallest permissible number in CM1). To each of these levels, identical 2D velocity fields associated with a given vortex patch configuration were assigned as initial condition. The simulations are technically "quasi-2D," but there practically is no difference in the solutions at the different model levels. The large time step of all simulations was $0.5 \mathrm{~s}$.

As there is no pressure perturbation added to the initial condition, acoustic adjustment occurs during the first time steps. At the same time, acoustic waves are emitted from the corners of the domain, presumably owing to weak but nonzero induced flow at the domain boundaries. ${ }^{2}$ These acoustic modes propagate back and forth through the domain but dissipate within a few minutes into the simulation. For this reason the pressure fields shown in the next section are plotted only after these acoustic waves are no longer noticeable.

In summary, several different vorticity distributions were specified and inverted to obtain the associated solenoidal velocity field. This flow field was then

\footnotetext{
${ }^{2}$ Adjusting the boundary conditions to allow for an initial condition with horizontally inhomogeneous flow, and thus possibly avoiding these acoustic modes, requires reformulation of the lateral boundary conditions, as discussed by Richardson et al. (2007).
}

allowed to evolve fully nonlinearly using the CM1 model. To gain an understanding of the axisymmetrization mechanism, the evolution of several vortex patches of increasing complexity, mimicking the patches observed in simulated supercells, are presented in the next section.

\section{Results}

\section{a. Kirchhoff elliptic vortex}

As a test of the method, we consider an elliptic vortex patch with uniform vorticity, also known as a Kirchhoff vortex (e.g., Wu et al. 2006, p. 285). This case is selected because there exists a simple analytical expression for the rotation rate of the ellipse. Just by virtue of its own induced flow, this vortex patch can be shown to rotate as a whole at a constant angular velocity of

$$
\Omega=\zeta \frac{a b}{(a+b)^{2}}
$$

where $\zeta$ is the constant vorticity in the ellipse, and $a$ and $b$ are the semiminor and semimajor axes, respectively. Here the major axis is initially aligned with the $y$ axis. For our test case $(a=1000 \mathrm{~m}, b=$ $5000 \mathrm{~m}$, and $\zeta=0.03 \mathrm{~s}^{-1}$ ), a theoretical angular velocity of $\Omega \approx 0.24^{\circ} \mathrm{s}^{-1}$ results. As seen in Fig. 1, this closely matches the simulation results (e.g., rotation by $108^{\circ}$ in $450 \mathrm{~s}$, Fig. 1c). The $W_{k}$ number remains approximately constant during the simulation, and indicates only a slight dominance of rotational motion over straining motion. The ellipse exhibits spiral filaments (right column of Fig. 1), probably resulting from finite vorticity gradients due to implicit diffusion as well as numerical dispersion, which leads to small negative vorticity extrema near the tips (not shown). Despite these epiphenomena, the overall shape of the ellipse remains intact and the method may thus confidently be applied to the other scenarios.

\section{b. Symmetric nonuniform vortex patch}

While the Kirchhoff vortex may be considered a highly idealized representation of a vertical-vorticity river, its uniform vorticity results in solid-body-like rotation of the entire ellipse. A better model of the near-ground vorticity distribution in supercells is an elliptic patch with vorticity maximized in the center of the patch. The patch used in this experiment features a Gaussian vorticity distribution in the $x$ and $y$ directions, with a maximum vertical vorticity 

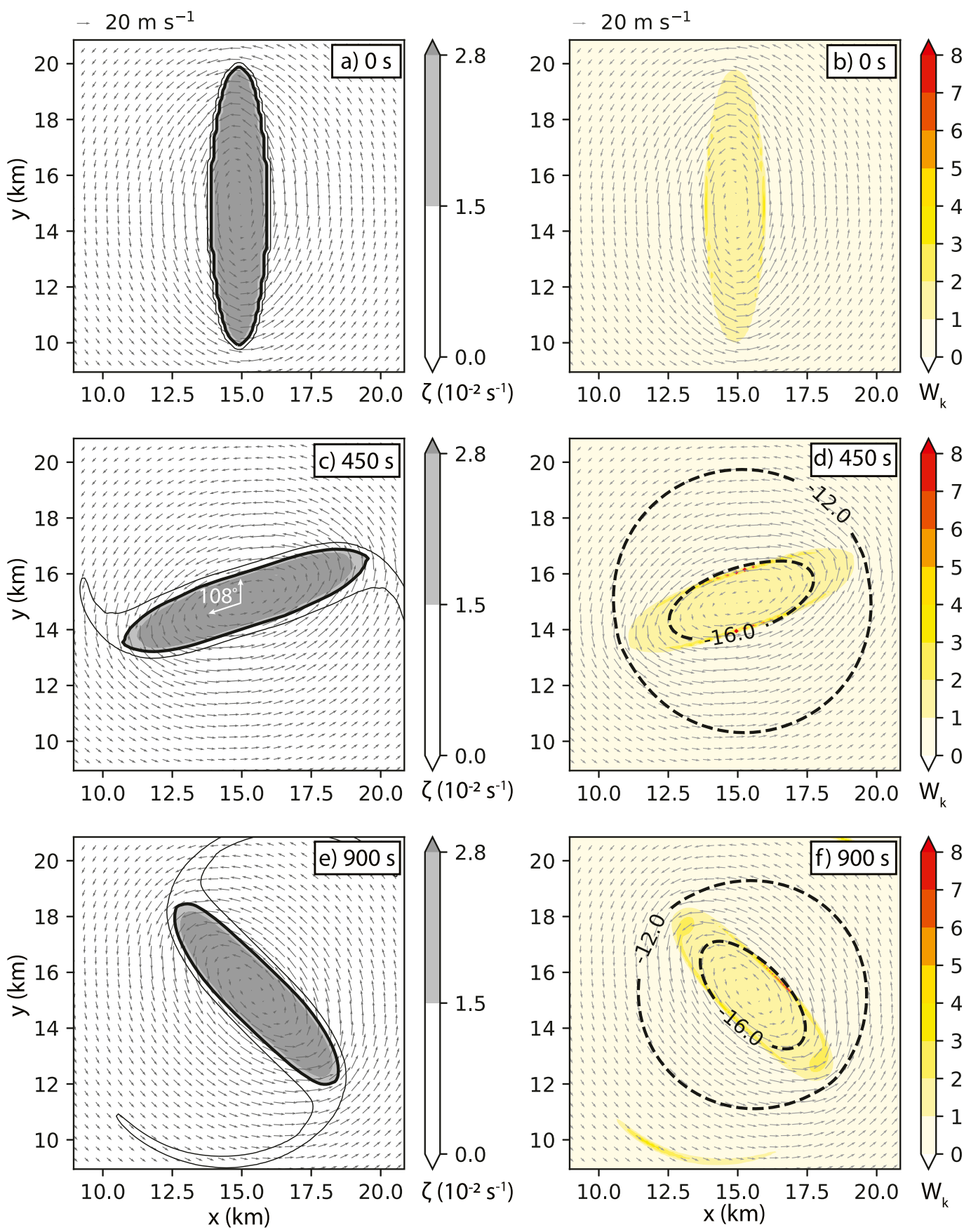

FIG. 1. Shown is the temporal evolution of the Kirchhoff elliptic vortex. (left) Vorticity (in $10^{-2} \mathrm{~s}^{-1}$ ) is shaded, and also contoured for $0.1 \times 10^{-2} \mathrm{~s}^{-1}$ (thin contour) and $1.5 \times 10^{-2} \mathrm{~s}^{-1}$ (thick contour). Contours for larger values were omitted because these failed to outline the ellipse cleanly due to numerical noise. (right) The kinematical vorticity number is shaded ( $W_{k}$, dimensionless) and the pressure perturbation is contoured in $4 \mathrm{hPa}$ intervals. The induced wind field is also plotted (every fourth vector is shown, scale vector at the top of the figure): (a),(b) initial condition; (c),(d) $450 \mathrm{~s}$; and (e),(f) $900 \mathrm{~s}$.

value of $\zeta=0.03 \mathrm{~s}^{-1}$ and the same geometry as in the previous experiment. In this case, the induced velocity field leads to differential advection of vorticity such that isolation of the central vorticity maximum results, as shown in Fig. 2. Although the velocity increases with increasing radius, the angular velocity of the outer portions of the ellipse is less than the angular velocity of the inner portions, 

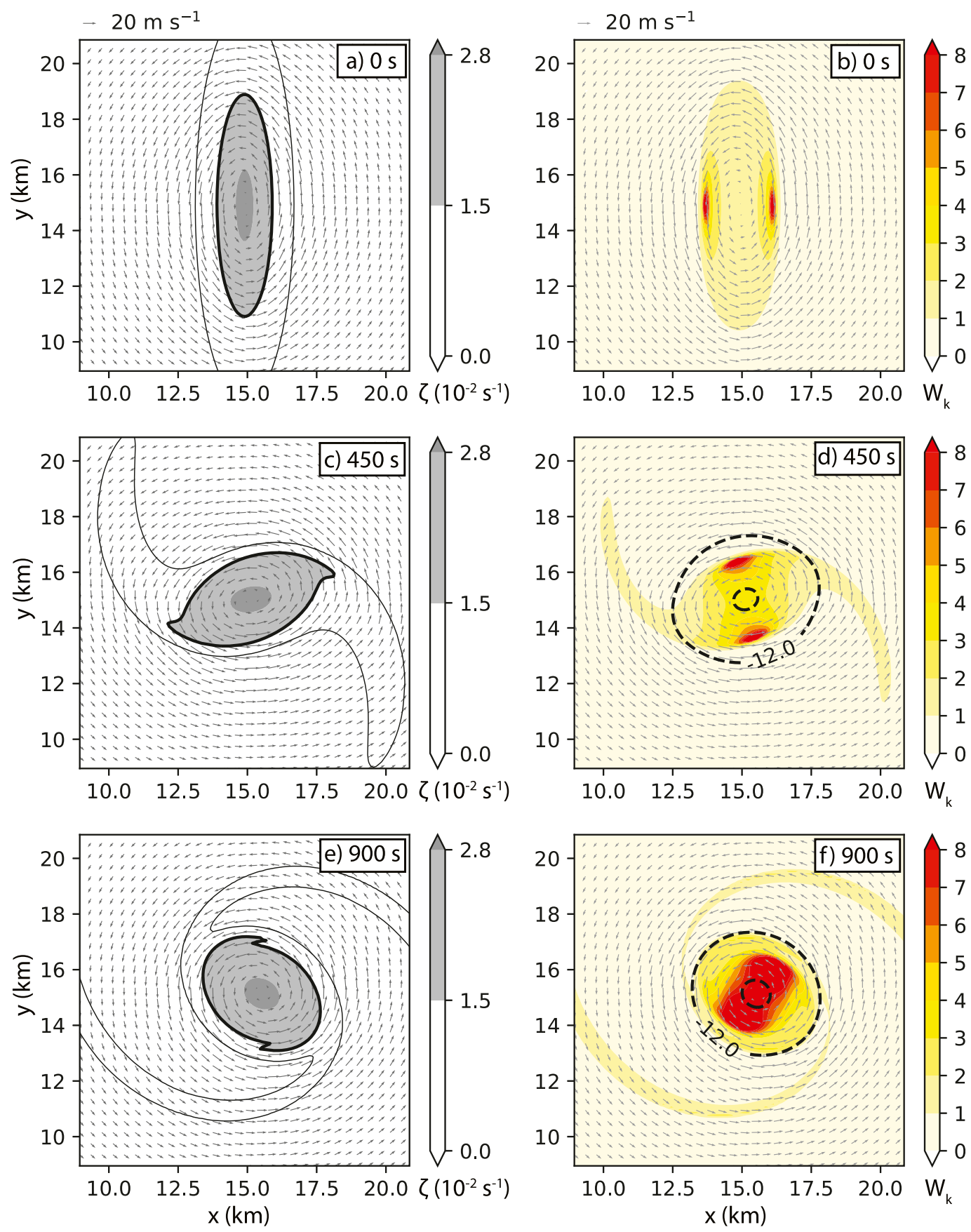

FIG. 2. Shown is the temporal evolution of a nonuniform elliptic vortex patch. (left) Vorticity (in $10^{-2} \mathrm{~s}^{-1}$ ) is shaded, and also contoured for $0.1 \times 10^{-2} \mathrm{~s}^{-1}$ (thin contour) and $1.5 \times 10^{-2} \mathrm{~s}^{-1}$ (thick contour). (right) The kinematical vorticity number is shaded ( $W_{k}$, dimensionless) and the pressure perturbation is contoured in $4 \mathrm{hPa}$ intervals. The induced wind field is also plotted (every fourth vector is shown, scale vector at the top of the figure): (a),(b) initial condition; (c),(d) $450 \mathrm{~s}$; and (e),(f) $900 \mathrm{~s}$.

leading to the formation of spiral bands, which are ultimately shed from the vortex core. This process has been studied in detail by Melander et al. (1987) and is a prime example of vortex-patch axisymmetrization. This evolution is reflected by an increase of the kinematical vorticity number $W_{k}$, which is accompanied by an increasing pressure deficit in the center of the developing vortex Figs. $2 \mathrm{~d}-\mathrm{f}$. The increase of $W_{k}$ is associated with a decreasing rate of deformation by almost an order of magnitude, starting from about $1.5 \times 10^{-2} \mathrm{~s}^{-1}$ (not shown). 


\section{c. Asymmetric nonuniform vortex patch}

The next vortex patch is initialized using a skewnormal vorticity distribution along the $y$ axis and a Gaussian distribution along the $x$ axis. This patch more closely resembles the typical configuration of a vertical-vorticity river (e.g., Fig. 1 of Gaudet et al. 2006). The skew-normal distribution is given by a product involving a Gaussian distribution and the error function:

$$
\zeta\left(x_{0}, y\right)=2 B f(y) F(y),
$$

where $x_{0}=15 \mathrm{~km}$ is the center of the patch in $x$ direction and

$$
f(y)=\frac{1}{\sigma \sqrt{2 \pi}} \exp \left[-\frac{1}{2}\left(\frac{y-y_{0}}{\sigma}\right)^{2}\right],
$$

and

$$
F(y)=\frac{1}{2}\left[1+\operatorname{erf}\left(\alpha \frac{y-y_{0}}{\sigma \sqrt{2}}\right)\right],
$$

where $\alpha$ is the skewness (set to 10.0 ), and $y_{0}=10 \mathrm{~km}$ is the location of the southern tip of the patch. The amplitude $B$ is given by

$$
B=1.33 \sigma \zeta_{\max },
$$

where $\sigma$ is the standard deviation of the Gaussian distribution (set to $8000 \mathrm{~m}$ ), $\zeta_{\max }=0.03 \mathrm{~s}^{-1}$ is the maximum vorticity in the patch, and the factor of 1.33 has been added so that the maximum of the distribution corresponds (approximately) to the $\zeta_{\max }$ value.

The time evolution of this patch is depicted in Fig. 3. Differential vorticity advection induced by the nonuniform vorticity distribution engenders a collapse of the river-like patch into a symmetric vortex, much like in the previous example. The kinematical vorticity number increases as vortex axisymmetrization progresses (Figs. 3d-f), highlighting the increasing dominance of rotational flow over straining motion. During the process, the core pressure perturbation decreases by about $6 \mathrm{hPa}$.

\section{d. Vortex patch from a $3 D$ supercell simulation}

Adding another layer of complexity, a vortex patch that developed in a full $3 \mathrm{D}$ supercell simulation is presented. The simulation uses the Weisman and Klemp thermodynamic profile (Weisman and Klemp 1982, in the following WK82) with a standard quarter-circle hodograph, a horizontal grid spacing of $200 \mathrm{~m}$, a freeslip lower boundary, and the Morrison et al. (2009) microphysics scheme. At 3720 s simulation time, an elongated, roughly elliptic vortex patch had developed, which subsequently evolved into a relatively intense vortex (Figs. 4a,c,e). The vorticity field at $3720 \mathrm{~s}$ was extracted and inverted as described in section 2. To ensure approximate fulfillment of the homogeneous Dirichlet boundary condition, the vorticity was relaxed to zero for all grid points at distances greater than $15 \mathrm{~km}$ away from the vortex patch of interest. The horizontal domain size was $75 \times 75 \mathrm{~km}^{2}$.

Following the same procedure as in the previous experiments, the induced flow field was fed into the 2D configuration of CM1 and allowed to evolve consistent with Eq. (7). The 2D simulation was run at the same horizontal grid spacing as the 3D simulation $(200 \mathrm{~m})$. The vorticity distribution (contoured) along with $W_{k}$ in the 3D simulation at $3720 \mathrm{~s}$ is shown in Fig. 4a. The initial vortex patch resembles the configuration presented in section $4 \mathrm{c}$ and is depicted in Figs. $4 \mathrm{a}$ and $4 \mathrm{~b} .{ }^{3}$ Both vortex patches collapse into a vortex as seen by the vorticity distribution as well as the increasing kinematical vorticity number (Fig. 4). The vortex in the 3D simulation (Figs. 4a,c,e) develops much faster than in the 2D setting (Figs. 4b,d,f), likely because the vortex is intensifying due to vertical stretching in the 3D simulation during axisymmetrization, leading to larger induced velocities (no stretching occurs in the $2 \mathrm{D}$ case). The peak vorticity in the 3D simulation reaches $0.1 \mathrm{~s}^{-1}$. Aside from the timing and intensity differences, the $2 \mathrm{D}$ setting does capture the overall development.

\section{e. Simulations with background strain}

Two experiments were run with a background strain field. The first scenario was realized by superposing a west-east-oriented vortex patch with a skew-Gaussian vorticity distribution to a large and highly elongated, south-north-oriented Kirchhoff vortex with $\zeta=0.01 \mathrm{~s}^{-1}$ and an aspect ratio of about 13 (Fig. 5a). The flow associated with this Kirchhoff ellipse is approximately a shear layer with strain

\footnotetext{
${ }^{3}$ By first cross differentiating the horizontal CM1 velocity components, and then inverting the vorticity to give the initial velocity distribution, some smoothing occurred. To ensure approximately the same initial vorticity peak in the $2 \mathrm{D}$ and 3D setups, the vorticity field taken from CM1 was increased by $40 \%$ before inverting it.
} 

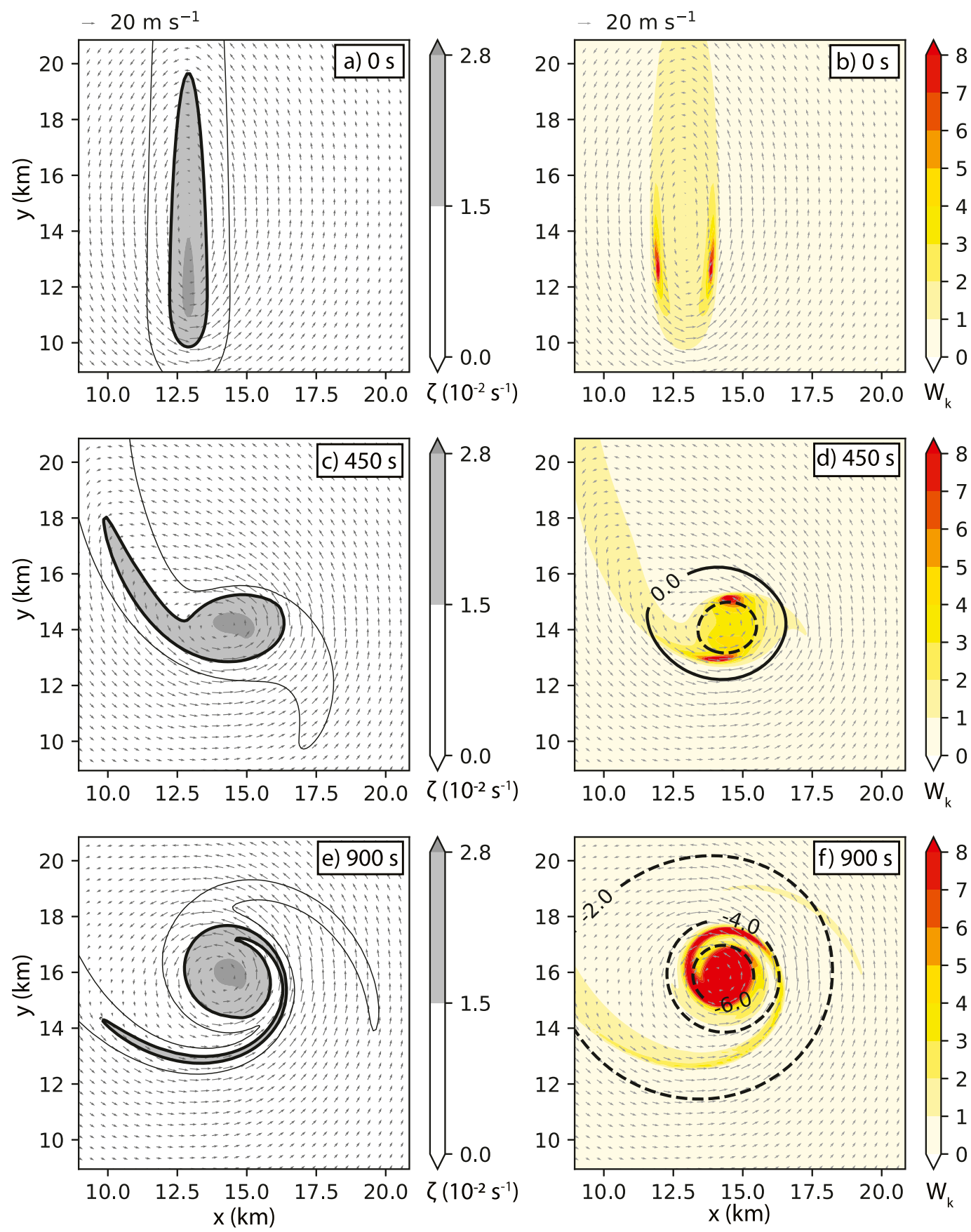

FIG. 3. Shown is the temporal evolution of a nonuniform vortex patch with skew-Gaussian vorticity distribution along the major axis. (left) Vorticity (in $10^{-2} \mathrm{~s}^{-1}$ ) is shaded, and also contoured for $0.1 \times 10^{-2} \mathrm{~s}^{-1}$ (thin contour) and $1.5 \times 10^{-2} \mathrm{~s}^{-1}$ (thick contour). (right) The kinematical vorticity number is shaded $\left(W_{k}\right.$, dimensionless) and the pressure perturbation is contoured in $2 \mathrm{hPa}$ intervals. The induced wind field is also plotted (every fourth vector is shown, scale vector at the top of the figure): (a),(b) initial condition; (c),(d), $450 \mathrm{~s}$; and (e),(f), $900 \mathrm{~s}$.

rates roughly matching the vorticity magnitude, and a dilatation axis oriented southwest-northeast. In the first experiment, the superposed patch of interest (resulting in a peak total vorticity of $0.02 \mathrm{~s}^{-1}$ ) is aligned such that its major axis is initially orthogonal to the shear vector, which initially points to the north along the symmetry axis. The patch quickly rotates counterclockwise toward the dilatation axis and 

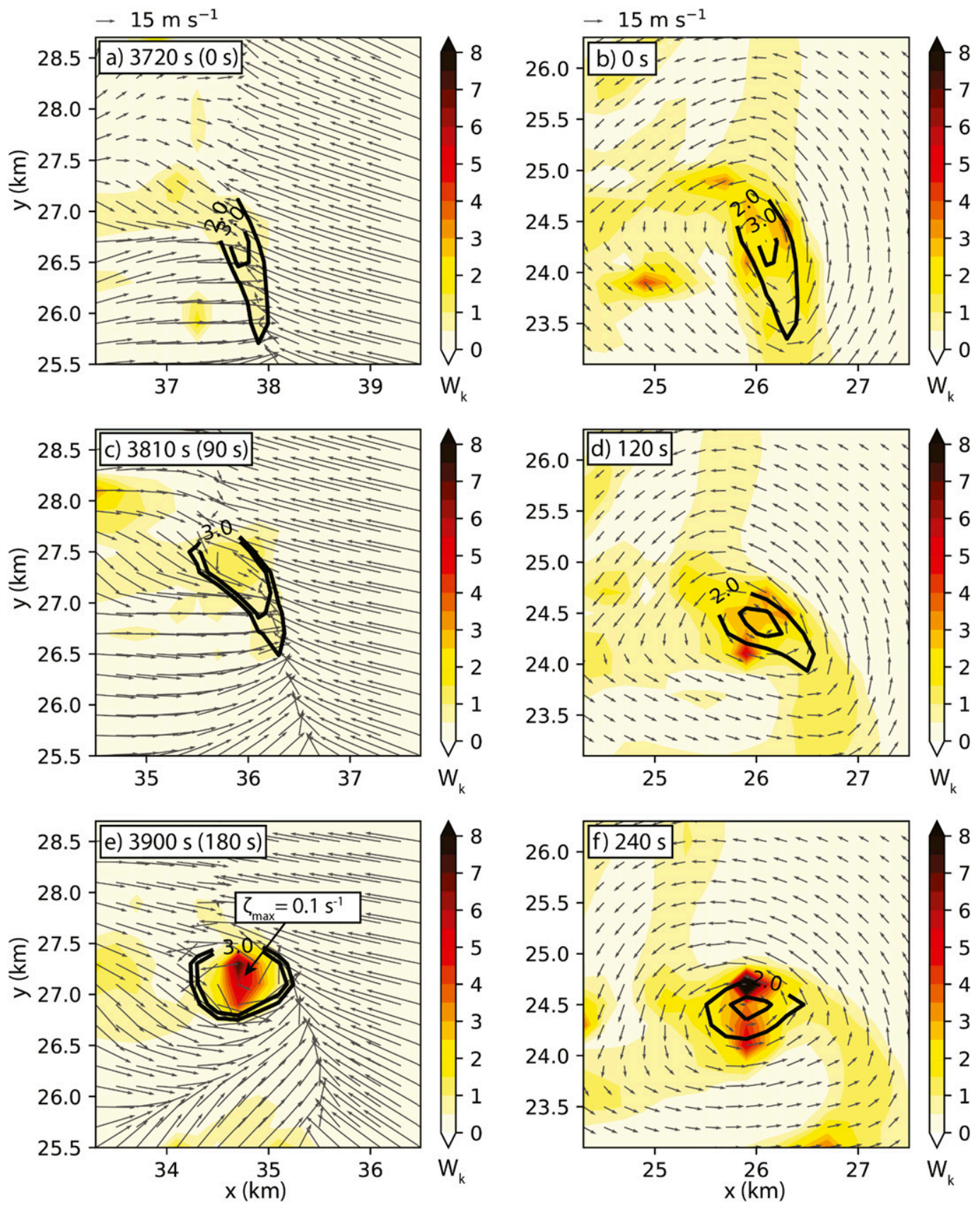

FIG. 4. Shown is the temporal evolution of the vorticity distribution taken from a 3D supercell simulation. (left) The evolution at the lowest model level of the WK82 simulation is shown, while (right) the evolution of the same vorticity distribution in the $2 \mathrm{D}$ framework is shown. Vorticity is contoured $\left[(2,3) \times 10^{-2} \mathrm{~s}^{-1}\right]$ and the kinematical vorticity number ( $W_{k}$, dimensionless) is shaded. Approximately vortex-relative wind vectors are also shown (scale vectors at the top of the figure). (a),(c),(e) The fields from the supercell simulation are shown for every $90 \mathrm{~s}$ simulation time, while (b),(d),(f) the fields from the $2 \mathrm{D}$ experiment are shown for every $120 \mathrm{~s}$ simulation time.

asymptotically aligns with the shear vector, while becoming increasingly elongated (Fig. 5b). Eventually, the ellipse disappears due to diffusion, and no vortex forms. Indeed, $W_{k}$ decreases from an initial value of about three (Fig. 5a) during the deformation of the patch. This is an axisymmetrization failure mode, in which the rate of deformation of the patch is much larger than the rate at which axisymmetrization occurs 

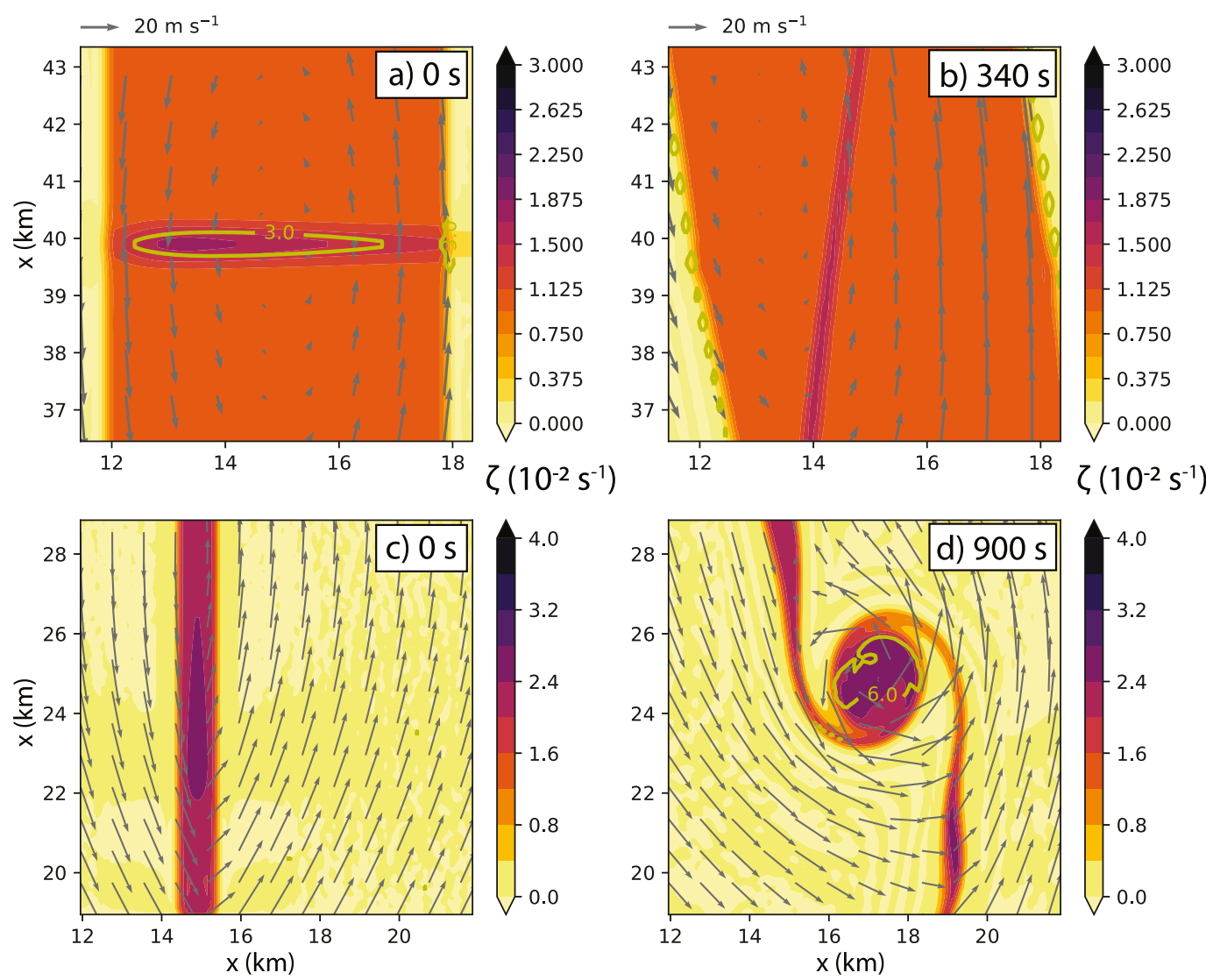

FIG. 5. Shown is the temporal evolution of a vortex patch embedded in a sheared flow. (a),(b) Vorticity (shaded), kinematical vorticity number (only one contour is shown in both panels, $W_{k}=3$ ), and velocity field (every eighth vector is shown, scale vector for all plots at the top of the figure) for (a) $0 \mathrm{~s}$ and (b) $340 \mathrm{~s}$. (c), (d) As in (a), (b), but the contour represents $W_{k}=6$ in both panels and for (c) $0 \mathrm{~s}$ and (d) $900 \mathrm{~s}$.

[consistent with the MTV $<\mathrm{CCV}$ case described by Gaudet et al. (2006)]. ${ }^{4}$

A more realistic setup in relation to the near-surface flow field of a supercell is modeled by a configuration in which the major axis of the vortex patch is initially parallel to the vorticity band (Fig. 5c). Here, the shear layer exhibits rather large deformation $(\approx$ vorticity $)$ of about $0.02 \mathrm{~s}^{-1}$ within the elliptic patch, which has an aspect ratio of 50 . With the superposed nonuniform vortex patch a peak total vorticity of $0.03 \mathrm{~s}^{-1}$ results. In this case the straining motion aids the axisymmetrization process by compressing the patch's major axis, leading to a relatively intense vortex, as shown in Fig. 5 d. The $W_{k}$ number increases to above six during vortex formation. Gaudet et al. (2006) likewise found that the background strain contributed to vortex formation

\footnotetext{
${ }^{4} \mathrm{MTV}$ is the maximum tangential velocity induced by the vortex patch and $\mathrm{CCV}$ is the characteristic background confluent velocity.
}

in their simulation. Interestingly, a kinematic structure arises that is reminiscent of the left-flank convergence boundary (LFCB; Beck and Weiss 2013) and rear-flank gust front. However, the flow does not contain horizontal convergence and it is not clear whether this is the main mechanism determining the gust front configuration in 3D simulations.

\section{f. Simulations with varying diffusion}

An interesting phenomenon can be observed if the ellipse is so narrow that it is only marginally resolved (semiminor axis of $200 \mathrm{~m}$ at $100 \mathrm{~m}$ grid spacing). Even if the vorticity within the vorticity band is initially homogeneous, numerical dispersion favoring especially $2 \Delta x$ waves results in subtle inhomogeneities within the vorticity band as seen in Fig. 6a. Some of these maxima coagulate and form sufficiently strong and large inhomogeneities, such that vortex formation around these seed inhomogeneities results (Figs. 6a,b). Upon adding some sixth-order explicit diffusion (kdiff6 $=0.1$ ) and repeating the experiment, the numerical noise is dampened 

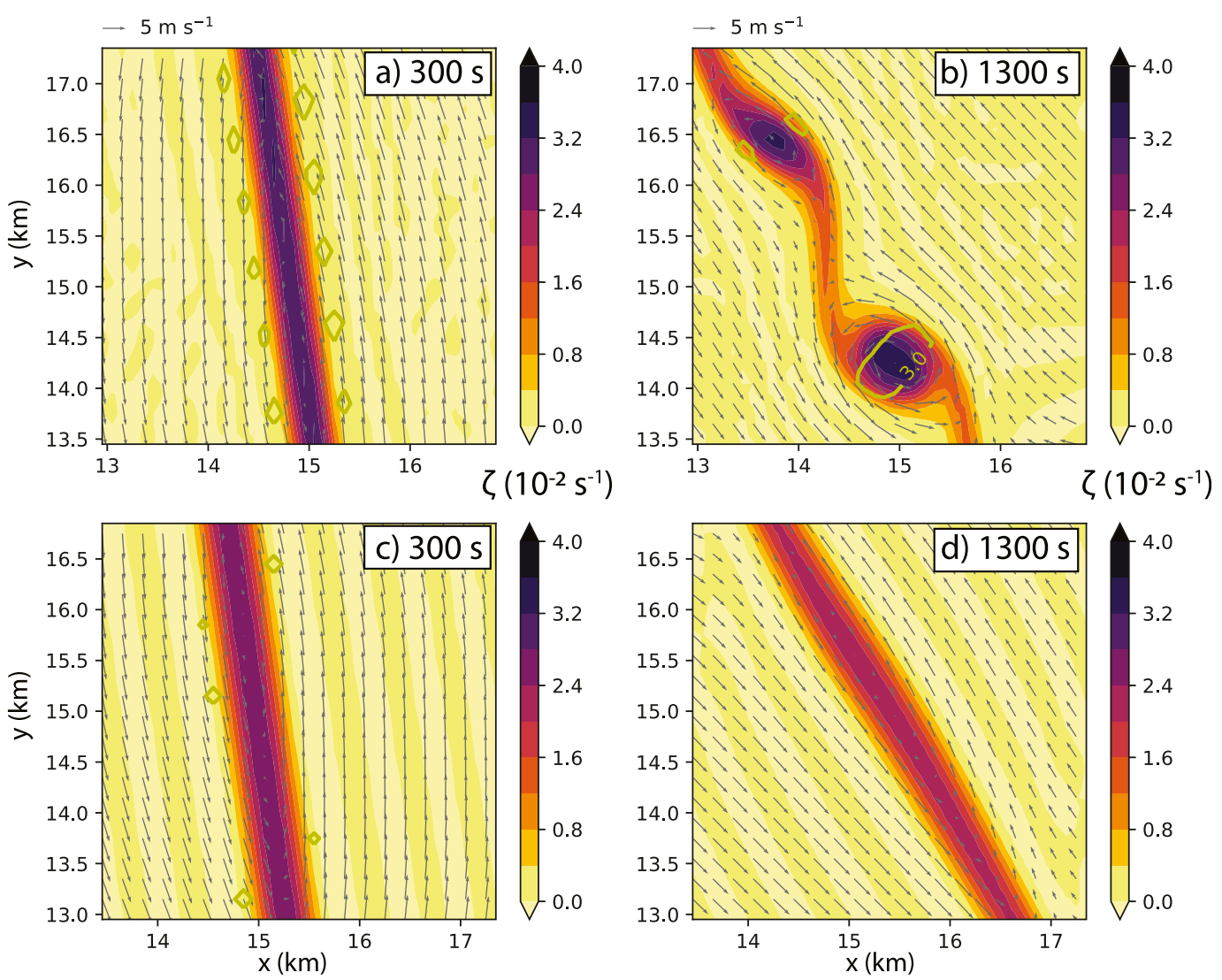

FIG. 6. Shown is the temporal evolution of a vortex patch with only implicit diffusion at (a) $300 \mathrm{~s}$ and (b) $1300 \mathrm{~s}$. (c),(d) The same vortex patch, but with added explicit diffusion. Shown in all panels are vorticity (shaded), the $W_{k}=$ 3 contour, and the induced velocity field (every second vector is shown, scale vector for all plots at the top of the figure).

and no axisymmetrization occurs, as shown in Figs. 6c and $6 \mathrm{~d}$. The reason for axisymmetrization failure in this case is that dampening proceeds at a faster rate than the axisymmetrization. Rerunning this scenario one more time, but without artificial diffusion and a horizontal grid spacing of $250 \mathrm{~m}$ instead of $100 \mathrm{~m}$, with the semiminor axis rescaled accordingly to $500 \mathrm{~m}$, results in a comparatively smooth solution without vortex formation in the central portions of the vorticity band (not shown). Certain choices of horizontal grid spacing as well as of the advection scheme and diffusion settings may thus lead to unphysical vortex formation in simulations.

\section{Discussion}

\section{a. Generality of the results}

The results imply that vortex-patch axisymmetrization (only) requires a nonuniform initial vorticity distribution. Since the goal of this study is to elucidate tornadogenesis, the focus is on nonuniform vortex patches, as those commonly occur within highresolution supercell simulations. However, there are instances in which vortex axisymmetrization occurs even with uniform vortex patches. One example is an undulating but otherwise homogeneous vorticity band with appropriate thickness and wavelength, which will lead to vortex formation after shear instability (e.g., Miles and Howard 1964) has been released. As discussed by, for example, Rayleigh (1880), Batchelor (2002), Hoskins et al. (1985), or Baines and Mitsudera (1994), the underlying physical mechanism of normalmode growth may still be understood in terms of self-induced motion due to vorticity extrema on either side of the vorticity band [these vorticity extrema are equivalent to PV anomalies, and the wave perturbations may be interpreted as barotropically unstable Rossby waves; see Hoskins et al. (1985), Lee and Wilhelmson (1997), or Gaudet et al. (2006)]. Stability of the band results when the perturbations are so small in scale that their induced flow is 
weaker than the background strain, as observed in section $4 \mathrm{e}$.

Besides the vorticity band, strained uniform elliptic vortex patches will also collapse given proper alignment relative to the dilatation axis, due to the mechanism described in section 4e [see also Neu (1984) or Wu et al. (2006), p. 285 ff.]. Even in the absence of background strain, amorphous but uniform vortex patches with the bulk of vorticity surrounding the center of mass may axisymmetrize as well, because differential selfadvection will tend to isolate the center and shed the smaller, peripheral features. All these examples are still consistent with Eq. (7) implying that symmetrization results from the rearrangement of the initial vorticity distribution via advection. However, the cases of homogeneous vortex patches do not seem to apply to vortex formation in supercells.

\section{b. Role of convergence and $3 D$ effects}

In 3D simulations, the vortex patches are usually tied to regions of enhanced horizontal convergence associated with internal surges traveling through the cold pool. Persistent, elongated patches are often associated with the horizontal convergence along the LFCB (Markowski et al. 2014). Although not addressed in this study, air is constantly rising through these patches. That is, at a given level parcels only spend a short amount of time in these features. This is unavoidable because horizontal convergence along the boundaries (and hence, accumulation of vertical vorticity) is associated with rising motion [note how the trajectories in Fig. 6 of Dahl et al. (2014) are rising after briefly having "dipped into" the vortex patch]. The role of the horizontal convergence is thus to give rise to, and shape, the vortex patches. As long as the horizontal convergence does not increase in time, a given vortex patch does not amplify. Consequently, at least the effect of time-independent background horizontal convergence is indirectly included in the experiments by virtue of the shape of the initial vorticity distribution. The 2D method captures the essence of vortex development in 3D because at a given level the $2 \mathrm{D}$ flow must still match the vorticity field (see also Gaudet et al. 2006).

In the context of a 3D setting, the processes discussed in this study do not eliminate the requirement for strong vertical stretching of near-ground vertical vorticity to achieve tornadogenesis, which depends on a combination of favorable ambient wind shear to support a strong mesocyclone in the lowest few $\mathrm{km}$, as well as adequate temperature deficits in the cold pool (e.g., Markowski and Richardson 2014; Coffer and Parker 2017; Coffer et al. 2017; Fischer and Dahl 2020, manuscript submitted to J. Atmos. Sci.). The axisymmetrization process itself only redistributes vorticity contours and hence cannot directly explain amplification of the vorticity. However, because the axisymmetrization is accompanied by decreasing core pressure, it appears that in 3D a feedback loop arises between vortex stretching/tilting and the vorticity-induced pressure deficit, as mentioned in the Introduction. Once a sufficiently strong pressure deficit is in place, the maintenance of the vortex no longer seems to rely solely on the DJB93 mechanism, but in addition near-surface horizontal vorticity generated baroclinically or frictionally may be utilized [without the detour via a downdraft; e.g., Rotunno et al. (2017); Roberts and Xue (2017)]. The axisymmetrization may thus help intensify the vortex and substantially modify its dynamics.

\section{c. Origin of the nonuniformities}

There is a combination of factors that gives rise to the nonuniformities that initiate the collapse of the vortex patch in $3 \mathrm{D}$ simulations. One factor is presented in Fig. $7 \mathrm{a}$, which is taken from the simulation presented in section $4 \mathrm{~d}$. The downdraft northwest of the vorticity band dispenses a carpet of near-surface positive vertical vorticity, which is focused along the LFCB due to horizontal convergence. However, this broad area of weak cyclonic vorticity is slightly inhomogeneous, which results in variations in vertical stretching along the convergence boundary. At the same time, the horizontal convergence, contoured in Fig. 7b, also varies along the boundary, which is related, for example, to downdraft unsteadiness, gust front orientation (Houston 2017), or proximity to the storm's updraft. A combination of these effects leads to nonuniform vertical vorticity amplification via stretching of vertical vorticity along the boundary. Incidentally, Fig. $7 \mathrm{~b}$ also shows some $2 \Delta x$ noise, which in this case leads to the unphysical collapse of the southern portion of the vortex patch (not shown).

\section{Conclusions}

Vortex development near the surface in simulated supercells often occurs as a more or less elongated vortex patch is axisymmetrized (i.e., "rolls up"). To investigate the underlying mechanism, the author conducted several 2D simulations of a variety of elliptic vortex patches. The results illustrate that vortex formation results from self-organization of the vortex patch due to differential horizontal advection of vorticity by the flow induced by vortex patch itself. For the vortex patches considered in this study, the only requirement for axisymmetrization is a vorticity distribution within the patch that exhibits finite-amplitude inhomogeneities resulting from nonmodal growth. The vortex condenses upon a local maximum in the vorticity distribution. A favorably aligned 

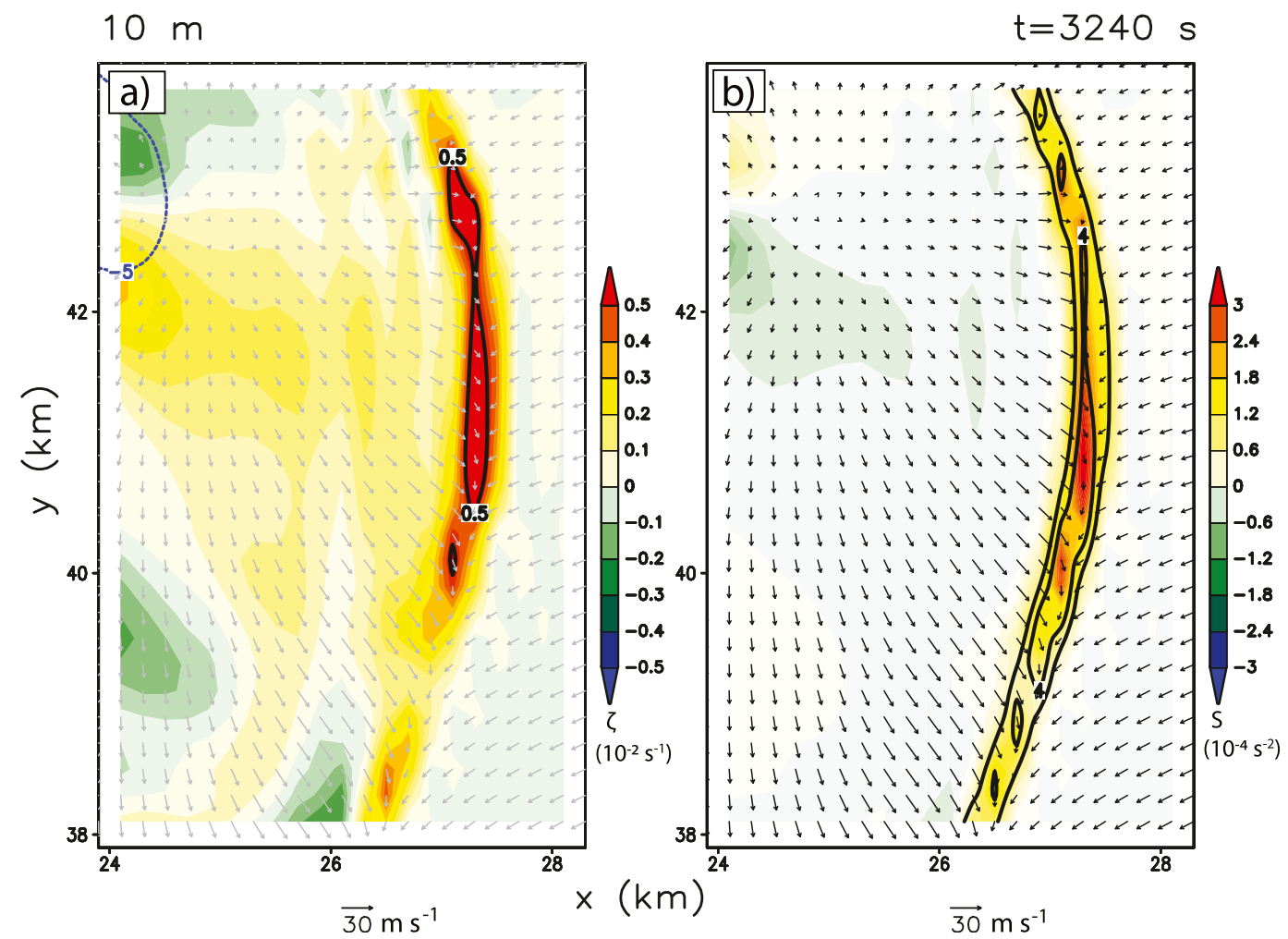

FIG. 7. (a) The storm-relative wind (vectors; scale at the bottom of the figure), vertical vorticity (shaded), as well as the $0.5 \times 10^{-2} \mathrm{~s}^{-1} \zeta$ contour at the lowest scalar model level $(10 \mathrm{~m} \mathrm{AGL})$. The $-5 \mathrm{~m} \mathrm{~s}^{-1}$ vertical-velocity contour at ca. $280 \mathrm{~m} \mathrm{AGL}$ is also shown (blue, dashed contour). (b) The stretching term of the vertical vorticity equation $\left[S=-\zeta\left(\nabla_{h} \cdot \mathbf{v}_{h}\right)\right.$; shaded] is plotted, as well as the horizontal convergence [contoured, for values of $(3,4) \times 10^{-2} \mathrm{~s}^{-1}$ ].

background strain field renders the axisymmetrization mechanism more efficient, supporting earlier results by Gaudet et al. (2006). Factors preventing axisymmetrization are relatively strong diffusion as well as background straining motion oriented such that it forces the ellipse to rotate quickly toward the symmetry plane of the background vorticity band. In these cases the time scale of selfadvection is larger than either the diffusive time scale or the advective time scale associated with the straining background flow, consistent with previous studies.

The results also show that the nonuniformity serving as seed for vortex formation may be introduced by numerical noise, implying that model settings such as the amount of diffusion (either implicit by choice of the order of the advection scheme, or explicit) or grid resolution may result in unphysical vortex formation on the scale of a few $\Delta x$.

Formally, the treatment adopted in this study is an initial value approach, which is distinct from the traditional treatment of shear instability along an infinitely extended vorticity band in terms of growing normal modes. With this approach the analysis is not limited to periodic perturbations, and the nonlinear evolution of arbitrary vortex patches may be analyzed.
Acknowledgments. Beneficial discussions especially early in the project with Drs. Chris Weiss and Eric Bruning are gratefully acknowledged. The author also thanks Drs. Brian Farrell, Gary Lackmann, Paul Markowski, Matt Parker, and Lisa Schielicke for sharing their thoughts on aspects of this study. Dr. George Bryan generously provided the source code for the CM1 model. Further, the author appreciates the reviews by Dr. Rich Rotunno and two anonymous reviewers, which helped clarify the presentation. This research was supported by NOAA under Grant NA15OAR4590226.

Data availability statement: Computer code and namelist settings are available from the author upon request.

\section{REFERENCES}

Baines, P. G., and H. Mitsudera, 1994: On the mechanism of shear flow instabilities. J. Fluid Mech., 276, 327-342, https://doi.org/ 10.1017/S0022112094002582.

Batchelor, G. K., 2002: An Introduction to Fluid Dynamics. Cambridge University Press, 615 pp.

Beck, J., and C. Weiss, 2013: An assessment of low-level baroclinity and vorticity within a simulated supercell. Mon. Wea. Rev., 141, 649-669, https://doi.org/10.1175/MWR-D-11-00115.1. 
Berger, S. A., 1988: Initial-value stability analysis of a liquid jet. J. Appl. Math., 48, 973-991, https://doi.org/10.1137/0148057.

Boyer, C., and J. M. L. Dahl, 2020: The mechanisms responsible for large near-surface surface vertical vorticity within simulated supercellular and quasi-linear storms. Mon. Wea. Rev., submitted.

Bryan, G. H., and J. M. Fritsch, 2002: A benchmark simulation for moist nonhydrostatic numerical models. Mon. Wea. Rev., 130, 2917-2928, https://doi.org/10.1175/1520-0493(2002)130<2917: ABSFMN $>2.0 . \mathrm{CO} ; 2$.

— , and H. Morrison, 2012: Sensitivity of a simulated squall line to horizontal resolution and parameterization of microphysics. Mon. Wea. Rev., 140, 202-225, https://doi.org/10.1175/MWRD-11-00046.1.

Case, K. M., 1960: Stability in inviscid plane Couette flow. Phys. Fluids, 3, 143, https://doi.org/10.1063/1.1706010.

Coffer, B. E., and M. D. Parker, 2017: Simulated supercells in nontornadic and tornadic VORTEX2 environments. Mon. Wea. Rev., 145, 149-180, https://doi.org/10.1175/MWR-D-16-0226.1.

, - J. M. L. Dahl, L. J. Wicker, and A. J. Clark, 2017: Volatility of tornadogenesis: An ensemble of simulated nontornadic and tornadic supercells in VORTEX2 environments. Mon. Wea. Rev., 145, 4605-4625, https://doi.org/10.1175/ MWR-D-17-0152.1.

Dahl, J. M. L., 2015: Near-ground rotation in simulated supercells: On the robustness of the baroclinic mechanism. Mon. Wea. Rev., 143, 4929-4942, https://doi.org/10.1175/MWR-D-15-0115.1.

, 2017: Tilting of horizontal shear vorticity and the development of updraft rotation in supercell thunderstorms. J. Atmos. Sci., 74, 2997-3020, https://doi.org/10.1175/JAS-D-17-0091.1.

— M. D. Parker, and L. J. Wicker, 2014: Imported and stormgenerated near-ground vertical vorticity in a simulated supercell. J. Atmos. Sci., 71, 3027-3051, https://doi.org/10.1175/ JAS-D-13-0123.1.

Davies-Jones, R. P. and H. E. Brooks, 1993: Mesocyclogenesis from a theoretical perspective. The Tornado: Its Structure, Dynamics, Prediction, and Hazards, Geophys. Monogr., Vol. 79, Amer. Geophys. Union, 105-114.

Drazin, P. G., and W. H. Reid, 1981: Hydrodynamic Stability. Cambridge University Press, 525 pp.

Farrell, B., 1982: The initial growth of disturbances in a baroclinic flow. J. Atmos. Sci., 39, 1663-1686, https://doi.org/10.1175/ 1520-0469(1982)039<1663:TIGODI > 2.0.CO;2.

__, 1984: Modal and non-modal baroclinic waves. J. Atmos. Sci., 41, 668-673, https://doi.org/10.1175/1520-0469(1984) 041<0668:MANMBW>2.0.CO;2.

—, 1987: Developing disturbances in shear. J. Atmos. Sci., 44, 2191-2199, https://doi.org/10.1175/1520-0469(1987)044<2191: DDIS $>2.0 . \mathrm{CO} ; 2$.

Fischer, J., and J. M. L. Dahl, 2020: The relative importance of updraft and cold pool characteristics on supercell tornadogenesis in highly idealized simulations. J. Atmos. Sci., submitted.

Flournoy, M. D., and M. C. Coniglio, 2019: Origins of vorticity in a simulated tornadic mesovortex observed during PECAN on 6 July 2015. Mon. Wea. Rev., 147, 107-134, https://doi.org/ 10.1175/MWR-D-18-0221.1.

Gaudet, B. J., and W. R. Cotton, 2006: Low-level mesocyclonic concentration by nonaxisymmetric transport. Part I: Supercell and mesocyclone evolution. J. Atmos. Sci., 63, 1113-1133, https://doi.org/10.1175/JAS3685.1.

—_ — - and M. T. Montgomery, 2006: Low-level mesocyclonic concentration by nonaxisymmetric transport. Part
II: Vorticity dynamics. J. Atmos. Sci., 63, 1134-1150, https:// doi.org/10.1175/JAS3579.1.

Hoskins, M. E., B. J. McIntyre, and A. W. Robertson, 1985: On the use and significance of isentropic potential vorticity maps. Quart. J. Roy. Meteor. Soc., 111, 877-946, https://doi.org/ 10.1002/qj.49711147002.

Houston, A., 2017: The possible role of density-current dynamics in the generation of low-level vertical vorticity in supercells. J. Atmos. Sci., 74, 3191-3208, https://doi.org/10.1175/JAS-D-16-0227.1.

Klemp, J. B., and R. Rotunno, 1983: A study of the tornadic region within a supercell thunderstorm. J. Atmos. Sci., 40, 359-377, https://doi.org/10.1175/1520-0469(1983)040<0359:ASOTTR $>$ 2.0.CO;2.

Lee, B. E., and R. B. Wilhelmson, 1997: The numerical simulation of non-supercell tornadogenesis. Part I: Initiation and evolution of pretornadic misocyclone circulations along a dry outflow boundary. J. Atmos. Sci., 54, 32-60, https://doi.org/ 10.1175/1520-0469(1997)054<0032:TNSONS $>2.0 . C O ; 2$.

Lewellen, D. C., and W. S. Lewellen, 2007: Near-surface intensification of tornado vortices. J. Atmos. Sci., 64, 2176-2194, https://doi.org/10.1175/JAS3965.1.

Lynch, P., 1988: Deducing the wind from vorticity and divergence. Mon. Wea. Rev., 116, 86-93, https://doi.org/10.1175/15200493(1988)116<0086:DTWFVA>2.0.CO;2.

__, 1989: Partitioning the wind field in a limited domain. Mon. Wea. Rev., 117, 1492-1500, https://doi.org/10.1175/15200493(1989) $117<1492$ :PTWIAL > 2.0.CO;2.

Markowski, P. M., and Y. P. Richardson, 2014: The influence of environmental low-Level shear and cold pools on tornadogenesis: Insights from idealized simulations. J. Atmos. Sci., 71, 243-275, https://doi.org/10.1175/JAS-D-13-0159.1.

__ — - M. Majcen, J. Marquis, and J. Wurman, 2011: Characteristics of the wind field in three nontornadic lowlevel mesocyclones observed by the Doppler on Wheels radars. Electron. J. Severe Storms Meteor., 6 (3), https:// www.ejssm.org/ojs/index.php/ejssm/article/viewArticle/75.

,-- , and G. Bryan, 2014: The origins of vortex sheets in a simulated supercell thunderstorm. Mon. Wea. Rev., 142, 39443954, https://doi.org/10.1175/MWR-D-14-00162.1.

Mashiko, W., 2016: A numerical study of the 6 May 2012 Tsukuba City supercell tornado. Part II: Mechanisms of tornadogenesis. Mon. Wea. Rev., 144, 3077-3098, https://doi.org/ 10.1175/MWR-D-15-0122.1.

Melander, M. V., J. C. McWilliams, and N. J. Zabusky, 1987: Axisymmetrization and vorticity gradient intensification of an isolated two-dimensional vortex through filamentation. J. Fluid Mech., 178, 137-159, https://doi.org/10.1017/S0022112087001150.

Miles, J. W., and L. N. Howard, 1964: Note on a heterogeneous shear flow. J. Fluid Mech., 20, 331-336, https://doi.org/10.1017/ S0022112064001252.

Morrison, H., G. Thompson, and V. Tatarskii, 2009: Impact of cloud microphysics on the development of trailing stratiform precipitation in a simulated squall line: Comparison of oneand two-moment schemes. Mon. Wea. Rev., 137, 991-1007, https://doi.org/10.1175/2008MWR2556.1.

Neu, J. C., 1984: The dynamics of a columnar vortex in an imposed strain. Phys. Fluids, 27, 2397-2402, https://doi.org/10.1063/1.864543.

Nowotarski, C. J., P. M. Markowski, Y. P. Richardson, and G. H. Bryan, 2015: Supercell low-level mesocyclones in simulations with a sheared convective boundary layer. Mon. Wea. Rev., 143, 272-297, https://doi.org/10.1175/MWR-D-14-00151.1.

Orf, L., R. Wilhelmson, B. Lee, C. Finley, and A. Houston, 2017: Evolution of a long-track violent tornado within a simulated 
supercell. Bull. Amer. Meteor. Soc., 98, 45-68, https://doi.org/ 10.1175/BAMS-D-15-00073.1.

Parker, P. D., and J. M. L. Dahl, 2015: Production of near-surface vertical vorticity by idealized downdrafts. Mon. Wea. Rev., 143, 2795-2816, https://doi.org/10.1175/MWR-D-14-00310.1.

Pedlosky, J., 1964: An inital value problem in the theory of baroclinic instability. Tellus, 16, 12-17, https://doi.org/10.3402/ tellusa.v16i1.8892.

Rayleigh, L., 1880: On the stability, or instability, of certain fluid motions. Proc. Lond. Math. Soc., XI, 57-70.

Richardson, Y. P., K. K. Droegemeier, and R. P. Davies-Jones, 2007: The influence of horizontal environmental variability on numerically simulated convective storms. Part I: Variations in shear. Mon. Wea. Rev., 135, 3429-3455, https://doi.org/10.1175/MWR3463.1.

Roberts, B., and M. Xue, 2017: The role of surface drag in mesocyclone intensification leading to tornadogenesis within an idealized supercell simulation. J. Atmos. Sci., 74, 3055-3077, https://doi.org/10.1175/JAS-D-16-0364.1.

Rotunno, R., and J. Klemp, 1985: On the rotation and propagation of simulated supercell thunderstorms. J. Atmos. Sci., $\mathbf{4 2}$, 271-292, https://doi.org/10.1175/1520-0469(1985)042<0271: OTRAPO $>2.0 . \mathrm{CO} ; 2$.

, P. M. Markowski, and G. H. Bryan, 2017: "Near-ground" vertical vorticity in supercell thunderstorm models. J. Atmos. Sci., 74, 1757-1766, https://doi.org/10.1175/JAS-D-16-0288.1.
Saffman, P. G., 1992: Vortex Dynamics. Cambridge University Press, $311 \mathrm{pp}$.

Schenkman, A. D., M. Xue, and M. Hu, 2014: Tornadogenesis in a high-resolution simulation of the 8 May 2003 Oklahoma City supercell. J. Atmos. Sci., 71, 130-154, https://doi.org/10.1175/ JAS-D-13-073.1.

Schielicke, L., P. Névir, and U. Ulbrich, 2016: Kinematic vorticity number-A tool for estimating vortex sizes and circulations. Tellus, 68A, 29464, https://doi.org/10.3402/ tellusa.v68.29464.

Tao, T., and T. Tamura, 2020: Numerical study of the 6 May 2012 Tsukuba supercell tornado: Vorticity sources responsible for tornadogenesis. Mon. Wea. Rev., 148, 1205-1228, https:// doi.org/10.1175/MWR-D-19-0095.1.

Truesdell, C., 1954: The Kinematics of Vorticity. Indiana University Press, $232 \mathrm{pp}$

Weisman, M., and J. Klemp, 1982: The dependence of numerically simulated convective storms on vertical wind shear and buoyancy. Mon. Wea. Rev., 110, 504-520, https://doi.org/ 10.1175/1520-0493(1982)110<0504:TDONSC >2.0.CO;2.

Wicker, L. J., and R. B. Wilhelmson, 1995: Simulation and analysis of tornado development and decay within a three-dimensional supercell thunderstorm. J. Atmos. Sci., 52, 2675-2703, https:// doi.org/10.1175/1520-0469(1995)052<2675:SAAOTD>2.0.CO;2.

Wu, J.-Z., H.-Y. Ma, and M.-D. Zhou, 2006: Vorticity and Vortex Dynamics. Springer, $776 \mathrm{pp}$. 